\title{
Complexity Measure based on Sensitivity Analysis Applied to an Intensive Care Unit system
}

Joao R. B. Paiva ( $\nabla$ jricardobpaiva@yahoo.com.br )

Federal University of Goias (UFG)

Viviane M. Gomes

Federal Institute of Goias (IFG)

Poliana S. Barbosa

Federal University of Ceara (UFC)

Fabiana R. Almeida

Federal Institute of Goias (IFG)

Gabriel A. Wainer

Carleton University (CU)

Flavio A. Gomes

Federal University of Goias (UFG)

Wesley P. Calixto

Federal University of Goias (UFG)

\section{Research Article}

Keywords: Complexity measure, sensitivity analysis, Intensive Care Unit system

Posted Date: September 9th, 2021

DOl: https://doi.org/10.21203/rs.3.rs-873064/v1

License: (c) (1) This work is licensed under a Creative Commons Attribution 4.0 International License.

Read Full License 


\title{
Complexity measure based on sensitivity analysis applied to an Intensive Care Unit system
}

\author{
João R. B. Paiva ${ }^{1,2, *}$, Viviane M. Gomes ${ }^{1,2,4}$, Poliana S. Barbosa ${ }^{3}$, Fabiana R. Almeida ${ }^{1}$, \\ Gabriel A. Wainer ${ }^{4}$, Flávio A. Gomes ${ }^{2}$, and Wesley P. Calixto ${ }^{1,2}$
}

${ }^{1}$ Studies and Researches in Science and Technology Group (GCITE), Federal Institute of Goias (IFG), 74130-012, Goiania, GO, Brazil.

${ }^{2}$ Electrical, Mechanical and Computer Engineering School (EMC), Federal University of Goias (UFG), 74605-010, Goiania, GO, Brazil.

${ }^{3}$ Department of Clinical Medicine (DMC), Federal University of Ceara (UFC), 60020-181, Fortaleza, CE, Brazil.

${ }^{4}$ Department of Systems and Computer Engineering (DSCE), Carleton University (CU), K1S 5B6, Ottawa, ON,

Canada.

*joao.paiva@ifg.edu.br

\section{ABSTRACT}

This work proposes a system complexity metric and its application to Intensive Care Unit (ICU) system. The methodology for applying said complexity metric comprises: (i) parameters sensitivity indices calculation, (ii) mapping connections dynamics between system components, and (iii) system's complexity calculation. After simulating the ICU computer model and using the proposed methodology, we obtained results regarding: number of admissions, number of patients in queue, length of stay, beds in use, ICU performance, and system complexity values (in regular or overloaded operation). As the number of patients in queue increased, the ICU system complexity also increased, indicating a need for policies to promote system robustness.

\section{Introduction}

In critical care, we usually need to connect diverse interdependent entities to build a cohesive system capable to provide health recovery to patients. Those characteristics make the Intensive Care Unit (ICU) a complex system whose behavior is determined by rules that may change according to different needs ${ }^{1-3}$ (for instance, the different medical protocols). From admission to minimum operating conditions, multidisciplinary teams work cooperatively and effectively to achieve goals related to critical or potentially seriously sick patients recovery. In addition to human resources, critical care units need various devices, equipment and medicine, and this represents a significant cost for hospital management. Measuring ICU complexity can be useful to understand the health system dynamics in intensive care context, and the impact of small changes of some variables.

To address critical care mechanisms complexity, we need to see health care reality as a set of heterogeneous components that act as a whole. According to Morin ${ }^{4}$, complexity is a tissue of heterogeneous constituents inseparably associated or the tissue of events, actions, interactions, feedback, determinations and accidents that make up our phenomenal world.

Nature itself is characterized by complex organization patterns that combine regularity and randomness in its structure and behavior $^{5,6}$. Recurring patterns are often found in nature's ever-changing configurations, and even limited number of rules or laws may produce complex structures, e.g. DNA consists of strings of the same four nucleotides, yet no two individuals are exactly alike. This characteristic is known as perpetual novelty and is present in most complex systems ${ }^{3}$. For some decades, several studies have been addressing complexity as the quality of being complex or as scientific field with several branches ${ }^{3,7-10}$. These studies identify that complex systems may have some of these characteristics: non-linearity, emergence, self-organization, diversity, interdependence, evolution, and perpetual novelty, among others.

In intensive care context, we focus on two aspects: diversity and interdependence. From materials to people, we may observe diversity of intensive care service in terms of devices, equipment, drugs, procedures and professionals (physicians, residents, nurses, physiotherapists and technicians). According to Page ${ }^{1}$, diversity may enhances robustness of complex system maintaining its functionality, in addition to drive innovation and productivity. However if one part strongly coupled with others is affected, the whole system may be compromised ${ }^{11,12}$.

By observing these aspects at the ICU environment, we may analyze: (i) the diversity based on resources needed to intensive care and (ii) the interdependence based on adverse events cases. In addition to the impact on patients physical integrity, adverse events result in increased health care costs due to longer hospital stays; they affect health professionals psychologically and 
undermine confidence in medical staff. These implications have led to studies on safety culture that ensures early identification and prevention of major groups of adverse events ${ }^{13,14}$.

According to Rafter et al. ${ }^{13}$, methods such as Global Trigger Tool and Harvard Medical Practice Study were developed to identify adverse events through systematic charts review. Although these methods have applicability and sensitivity, the review process lack of standardization generates differences in obtained results, which makes it difficult to report. Despite the lack of reliable information on adverse events, studies estimate that between $4 \%$ and $17 \%$ of hospital admissions are associated with adverse events, and one to two thirds of these are preventable.

Research developed by Rothschild et al. revealed that $45 \%$ of adverse events recorded in a university tertiary hospital ICU were preventable, encompassing 223 serious errors, of which 11 were considered potentially fatal. These results reinforce the risks associated with critical care and the greater probability of adverse events in this environment. Therefore the careful assessment of preventable intercurrences profile is needed to act directly in prevention.

Forster et al. ${ }^{15}$ investigated whether adverse events are associated with length of stay at ICU and mortality. Adverse events were observed in $19 \%$ of ICU patients and refer to (i) procedural complication, (ii) nosocomial infection, (iii) adverse drug event, (iv) surgical complication, (v) therapeutic error, (vi) system error, and (vii) diagnostic error. The number of preventable events was $6 / 18,8 / 13,2 / 12,0 / 6,5 / 5,1 / 1$ and 1/1, respectively. As research findings, adverse events were independently associated with an average increase in hospital length of stay of 31 days, however a significant statistical association of adverse events and mortality was not found.

As length of stay at the ICU increased, the number of available beds decreased, which affects new patients admission. Using queuing theory and sensitivity analysis, McManus et al. ${ }^{16}$ assessed the impact of bed unavailability on ICU performance in a pediatric hospital during a 2 year period. Based on the admission, discharge and turn-away data, the authors observed that rejection rate increased exponentially as ICU utilization exceeded $80 \%$. This behavior was confirmed from results given by the queuing model, which also allowed to predict system performance from changes in unit size.

The unavailability of ICU beds obstructs entry flow, generating queues and even deaths due to delayed admission. Cardoso et al. ${ }^{17}$ analyzed outcomes of an ICU during 12 months: from 401 admissions, 125 patients were immediately admitted and 276 patients had delayed admission, resulting in 47 (37.6\%) and 138 (50\%) non-survivors, respectively. The authors verified that each waiting hour was independently associated with a 1.5\% increased death risk at ICU. Another study, developed by Town et al. ${ }^{18}$, verified the relationship between ICU readmission rates and ward cardiac arrest per 12-hour shift and available ICU beds at each shift start. The authors used data collected between 2009 and 2011 at a tertiary care academic medical center with 63 adult ICU beds and 272 adult general inpatient ward beds. As ICU bed availability decreased, readmission rate to the ICU within twenty-four hours and cardiac arrest rate among all ward patients increased.

The ICU system overload makes critical care environment more susceptible to errors or negligence, requiring greater staff attention and cooperation. Based on a game-theoretic experiment, Guazzini et al. ${ }^{19}$ verified that humans accurately estimate the benefits of collaboration when facing hard problems. Thus the staff behavioral responses in ICU environment may change according to the situation complexity.

Although there are studies on the intensive care complexity $2,20,21$, none of them applies complexity metrics and sensitivity analysis at the same time. Considering the restricted number of resources in intensive care, stay extension at the ICU due to adverse events, financial costs for the heath system, psychological and physical problems associated to adverse events or beds unavailability, this paper presents quantitative study on ICU complexity and applies sensitivity analysis for defining relevance of connections between elements. We propose a complexity metric that considers both internal and external factors, measuring the impact generated on outputs due to parameters variation, such as resources and patient arrival rate, in order to use sensitivity indices as connections weights.

The rest of the paper addresses the complexity of intensive care system of adult patients. We propose a computer-based model of intensive care units based on literature data, and a simulated flow that includes the patient arrival, resource demand, patient stay at ICU until discharge, and adverse events occurrence. The underlying disease and presence of comorbidities were not addressed in this paper, since each patient is seen in the simulation process as a person requiring intensive care regardless of what brought them to the ICU. In the next section, the theoretical framework is presented. Then, in the methodology section, the proposed complexity metric and the developed ICU modeling are presented. The results obtained from simulations of the ICU computational model are presented and discussed in the next sections, leading to some conclusions and proposed future work presented in the last section.

\section{Theoretical background}

This section presents theoretical bases necessary to understand the proposed methodology and obtained results. The concepts of (i) systems and models, (ii) system complexity, and (iii) sensitivity analysis are briefly described. 


\section{Systems, models and simulation}

Systems are composed of units that interact with each other through connections and with the environment through boundary components. Connectivity allows materials, energy and messages to flow. These units or system components working together achieve results that could not be obtained by individual components ${ }^{22-24}$. According to the systemness principle defined by Systems Science ${ }^{12,25}$, systems have the quality of encompassing subsystems and being encompassed by larger systems at the same time. Thus, systems are differentiated by their properties, which are: (i) totality - the internal cohesion among elements and boundaries components, (ii) composition - the elements and their interactions, (iii) internal organization - the way its structure performs functions, and (iv) external organization - the interaction rules with the environment ${ }^{12,25,26}$.

System modeling is the process of representing the system through behavior rules and interaction with the environment. Several studies and analysis can be performed using the model, to avoid costs, risks, or inconvenience of manipulating the real system ${ }^{27}$. During the modeling process, it is necessary to define which elements and interactions will be considered. The abstraction must consider all details necessary for the study, without addressing irrelevant particularities. The system model is the simplified representation of the real system, which can be built by different modeling techniques. The choice of technique depends on the modeler's experience and research emphasis ${ }^{28}$.

One of the techniques used to model real systems is Discrete Event System (DES) modeling. This technique refers to the class of systems that depend on events occurrence to evolve. The events are the result of intentional actions (spontaneous occurrence) or of actions triggered upon verification of a certain condition. These actions promote system state changes at random time intervals ${ }^{29}$. Both the events and states that describe discrete event systems can be mapped into finite discrete sets in the modeling process ${ }^{28}$.

Using the model, input parameters can be manipulated during experiments. Based on chosen scenarios, historical (real values) or hypothetical parameters values are applied to the model to observe the outputs ${ }^{29}$. These experiments can be performed using simulation, which aims to reproduce the modeled system dynamics behavior ${ }^{30}$. Thus, the output analysis allows to understand the system's functioning and to define strategies to improve its operation.

\section{System's complexity metrics}

Systems complexity comprises the system as an interacting whole (the parts interacting with each other and the whole interacting with the environment). Complex behaviors can be generated by simple mechanisms with simple interactions, as in the game of chess, in which different strategies and patterns emerge from a few rules and elements ${ }^{31}$. From a behavioral perspective, complexity is a subjective matter, since the system's behaviors are described by those who observe the system ${ }^{32,33}$.

Complex systems have the following characteristics: (i) nonlinearity - the superposition property absence, since only linear relations are represented by partial processes that can be superimposed to obtain the total process ${ }^{22}$, (ii) emergence - the quality of systems related to collective behavior, it is difficult to predict or explain ${ }^{8,34}$, (iii) self-organization - the ability to adjust its operation to establish its own rules and generate new behavior patterns without external intervention ${ }^{1,35,36}$ (iv) diversity heterogeneity of its member agents, allowing for cooperative relations ${ }^{1},(\mathrm{v})$ interdependence - the level of influence existing between system parts and the whole ${ }^{11}$, and (vi) evolution - the ability to develop trajectory in space and time by recombining structures that compose the system ${ }^{3}$.

The literature presents several complexity metrics, which are used to compare different systems of same nature or different configurations for the same system. Deacon and Koutroufinis ${ }^{5}$ and Gell-Mann ${ }^{37}$ propose that complexity consists of the balance between order and disorder, regularity and randomness. Thus, the ideal complexity metric considers completely random and completely ordered organizations as low complexity and intermediate organizations as high complexity. Considering the system under study, Lloyd ${ }^{38}$ lists forty system complexity metrics in three groups: (i) description difficulty, (ii) creation difficulty, and (iii) degree of organization. Complexity metrics can be based on entropy ${ }^{39}$, degree of hierarchy ${ }^{10}$, algorithmic information content $^{40,41}$, computational capacity ${ }^{42}$, thermodynamic depth ${ }^{43}$, statistics ${ }^{44}$, fractal dimension ${ }^{45}$, logical depth ${ }^{46}$, dynamic depth $^{5}$, amount of information ${ }^{34}$, size $^{8}$, and connections ${ }^{47}$.

\section{Sensitivity analysis}

Sensitivity analysis allows measuring the impact generated on system outputs due to variations in input variables ${ }^{48-50}$. The results obtained by the sensitivity analysis are used to: (i) indicate the need to simplify the model built to represent the system, (ii) investigate errors cause, (iii) verify how sensitive the system is to variations based on the relationship between input and output variables, (iv) confirm the correct processing of input parameters, (v) fix input variables that are not relevant, and (vi) identify regions of interest with regard to complexity and stability of the system ${ }^{50-52}$. Figure 1 , adapted from Gomes $(2020)^{53}$, illustrates the sensitivity analysis process for a system with $n$ input parameters given by $x_{1}, x_{2}, \cdots, x_{i}, \cdots x_{n}$.

Using local sensitivity analysis, we first investigate the parameters reference values that drive the system to its best performance. Thus, we define these reference values set, called the base case $\vec{\beta}$, given by: 


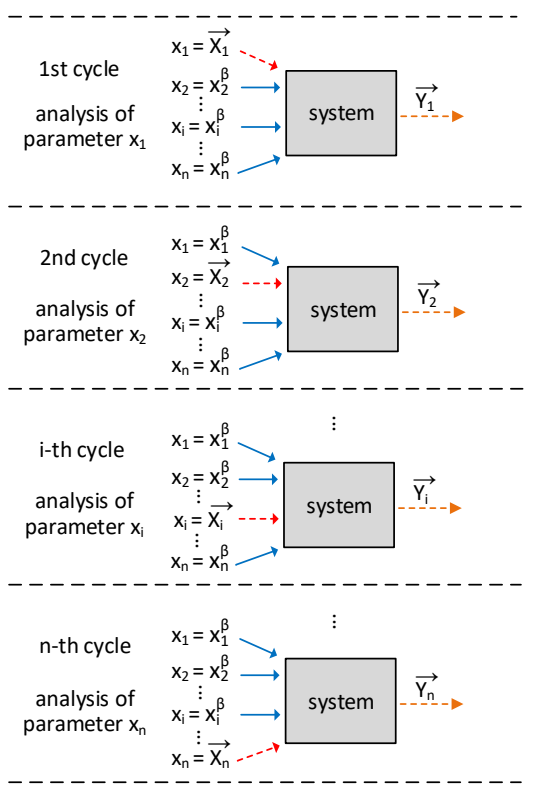

Figure 1. Sensitivity analysis cycles using the one-at-a-time method.

$$
\vec{\beta}=\left[x_{1}^{\beta}, x_{2}^{\beta}, \cdots, x_{i}^{\beta}, \cdots, x_{n}^{\beta}\right]
$$

where $x_{i}^{\beta}$ is the reference value for $x_{i}$ parameter. After defining the parameter base value, we must choose a set of values for each parameter in the range between $-100 \%$ and $100 \%$ from the base value. In Figure 1, we can see that the analysis cycle is performed for each system parameter. While modifying one parameter (red arrow), other are kept constant (blue arrows) in their reference values Thus, we can check the system's sensitivity to each $x_{i}$ parameter variations. This analysis comprises scenarios definition, that is, different values of the parameter under analysis in the variation range combined with the reference values of other parameters. These scenarios are simulated (or applied to the real system) and the obtained outputs are stored in $\overrightarrow{Y_{i}}$.

Among sensitivity analysis methods ${ }^{48,50,52,54}$, Eschenbach and McKeague ${ }^{55}$ propose a visual method using a graph called spider diagram. Figure 2, adapted from Pannell ${ }^{51}$, illustrates a spider diagram considering system sensitivity analysis with three input parameters: $x_{1}, x_{2}$ and $x_{3}$. Figure 2 shows the output values in the ordinate axis, the input parameters variation from the base value in the abscissa axis and, the ordered pairs corresponding to analyzed scenarios using markers.

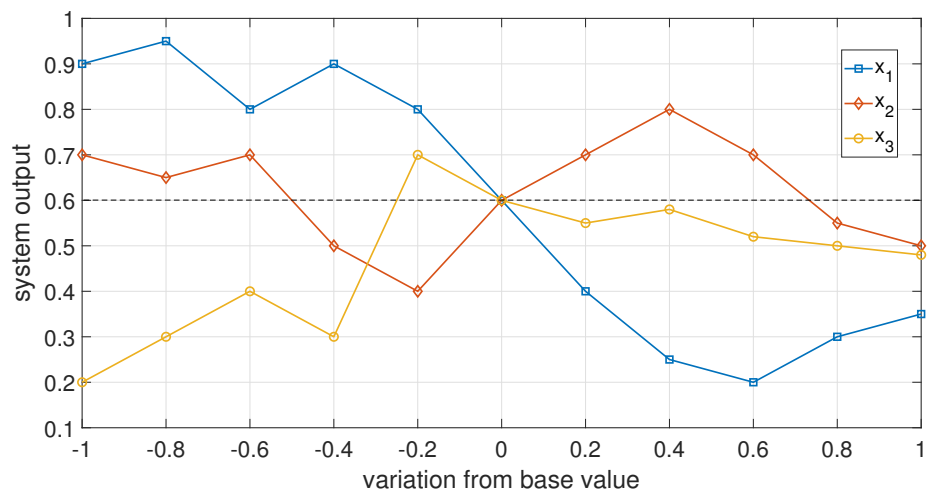

Figure 2. Hypothetical spider-diagram of the system sensitivity analysis with three input parameters.

In the spider diagram illustrated in Figure 2, all parameters can be modified in the range of $-100 \%$ to $100 \%$ from the base value given by $\vec{\beta}$. However, in practical situations, some parameters may have physical limitations that restrict this 
variation range. Even with such restrictions, the parameter can have high sensitivity. The more sensitive the parameter, the greater its impact on the system output (the greater the difference between obtained output values concerning the output value corresponding to $\vec{\beta}$ scenario). Based on this statement, Gomes ${ }^{56}$ proposes a method called the area method to obtain the sensitivity indices of input parameters. The spider diagram analysis interval is defined by the analyst, considering possible parameter restrictions.

The area method consists of the relationship between areas defined by the parameter curves. The axis parallel to the abscissa axis in the spider diagram is called the base axis. Thus, the areas between each parameter curve and the base axis correspond to the parameter contribution in relation to the total area. The parameter's sensitivity index is given by:

$$
S_{x_{i}}^{a}=\frac{A_{x_{i}}}{\sum_{i=1}^{n} A_{x_{i}}}
$$

where $S_{x_{i}}^{a}$ is the sensitivity index of $x_{i}$ parameter, $A_{x_{i}}$ is the area formed by the variation curve of $x_{i}$ parameter and the base axis, $n$ is the number of input parameters ${ }^{53}$.

\section{Methodology}

This section presents the methodology for calculating system complexity using modeling and simulation. We also present the intensive care unit (ICU) model for applying sensitivity analysis and complexity calculation.

\section{Proposed complexity metric}

The proposed complexity metric uses sensitivity indices to calculate complexity. These indices are used as connections weights of the system under study. Figure 3 illustrates the steps to apply the proposed metric. Based on the system model, we carry out the pre-analysis, when we define: (i) base scenario or base case, (ii) parameters variation range, and (iii) analysis interval in the spider diagram.

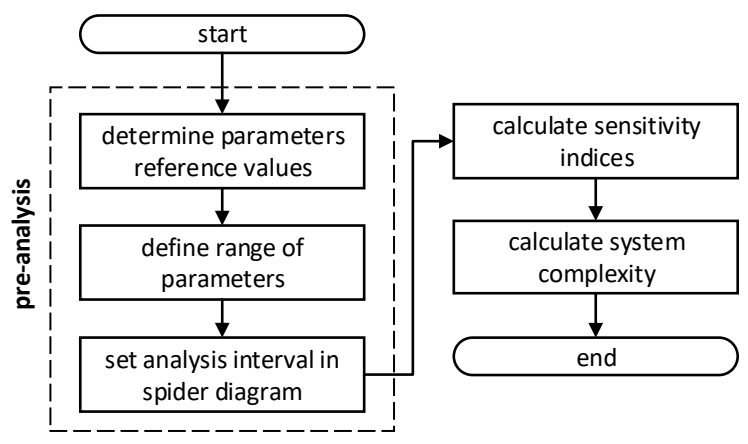

Figure 3. Proposed methodology action flow.

Using the area method, we can calculate the system parameters sensitivity indices, according to expression $(2)^{56}$. The output variable can be defined as the system performance. Considering modifying the reference values using one-at-a-time method, we can get performance values corresponding to different scenarios. Thus, the parameters can be related to the different system connections through sensitivity indices. For example, the connection between components $A$ and $B$ can be related to parameters $x_{2}, x_{3}$ and $x_{5}$. Therefore, this connection relevance $\gamma_{c}$ would be equal to the sum of these parameters sensitivity indices $\left(S_{x_{2}}^{a}+S_{x_{3}}^{a}+S_{x_{5}}^{a}\right)$. Finally, we propose that the system complexity is calculated by:

$$
\psi(c, \gamma)=\sum_{i=1}^{\rho}\left[\gamma_{c_{i}}-P\left(c_{i}\right) \cdot \log _{2} P\left(c_{i}\right)\right]
$$

where $\psi(c, \gamma)$ is the system complexity based on weighted connections, $\rho$ is the number of active connections at each instant, $P\left(c_{i}\right)$ is the connection probability $c_{i}$ to occur, and $\gamma_{c_{i}}$ is the connection relevance $c_{i}$.

The connection occurrence probability $P\left(c_{i}\right)$ can be theoretical or experimental, according to the system analysis. The number of active connections $\rho$ is given during system operation, where the expression (3) is calculated at each instant. The connection relevance $\gamma_{c_{i}}$ is obtained by the sum of parameters sensitivity indices directly related to the connection $c_{i}$. 


\section{Intensive Care Unit model}

The intensive care unit proposed model is defined based on the ICU patients flow. In summary, the model consists of the patients arrival, demand for resources, patients permanence in the ICU, adverse events occurrence, and patients exit after being discharged or dying. These relationships can be modeled as connections between system elements, as illustrated in Figure 4.

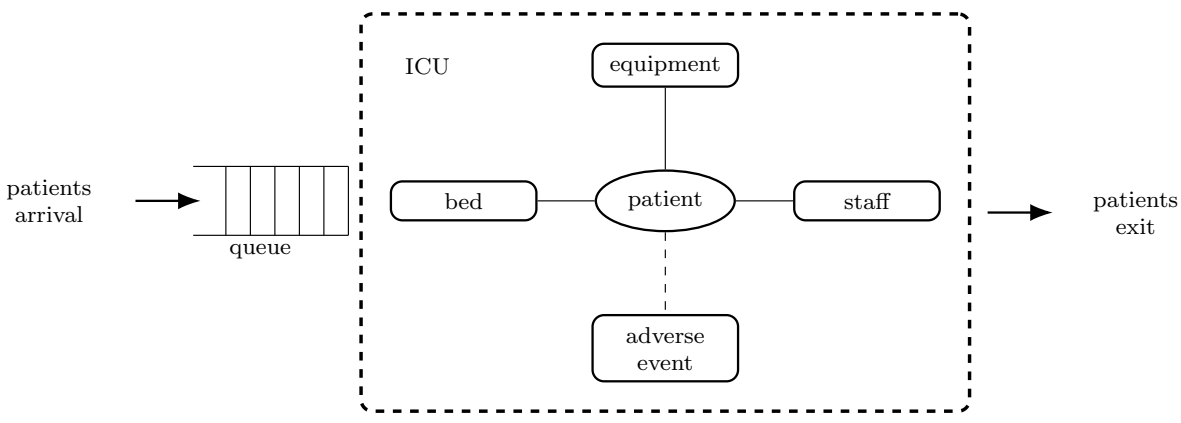

Figure 4. Connection-based ICU model.

The ICU patient flow starts by requesting an ICU bed. The patient waits in queue until resources are available and the intensivist physician confirms his/her admission. If the patient is admitted, resources are allocated, the patient occupies an ICU bed and receives care from staff. If the patient is not admitted, the bed request is canceled. In the case of hospitalization, the length of stay in ICU is increased whenever an adverse event occurs. During his/her stay in ICU, the patient can die. When the patient is discharged or dies, the resources are deallocated and the patient flow ends, as shown in Figure 5.

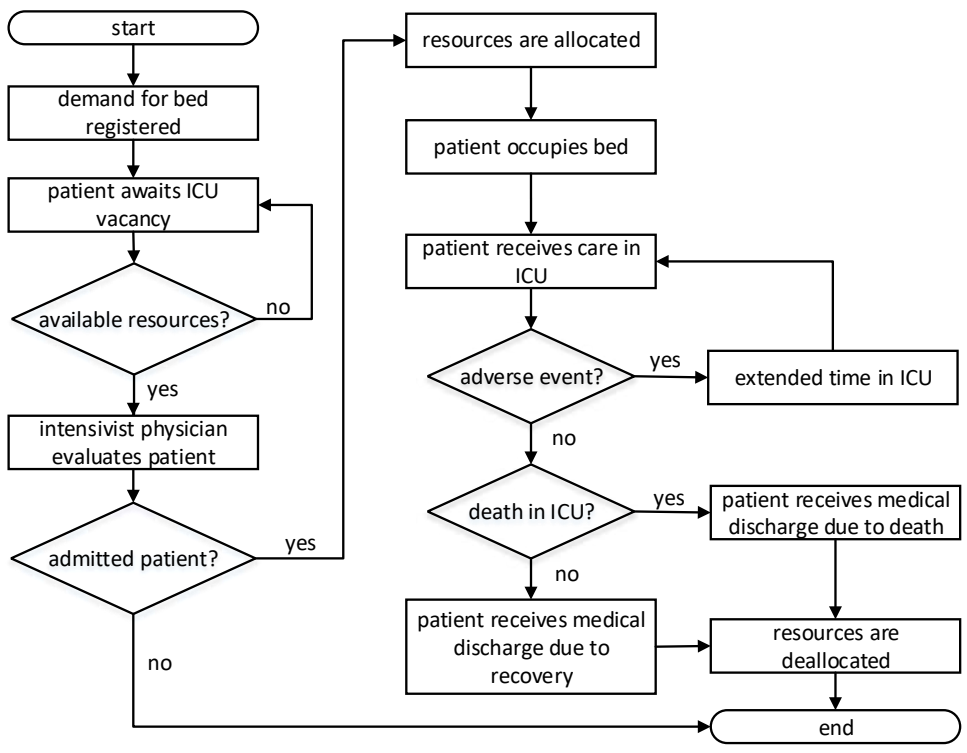

Figure 5. ICU Patient Flow.

Considering the ICU patient flow shown in Figure 5, the system dynamics are defined according to probability distributions. The system can be expressed in terms of states and events to perform system simulation of discrete events. The set of discrete states of patients in intensive care unit is: (i) in queue waiting for admission, (ii) in consultation with an intensivist physician, (iii) refused admission, (iv) hospitalized, (v) recovered, and (vi) deceased. The set of events is: (i) patient arrival (registration of vacancy demand), (ii) evaluation of patient's clinical status, (iii) refusal of admission, (iv) admission, (v) allocation of resources, (vi) occurrence of adverse event, (vii) death, (viii) patient exit (discharged patient or deceased patient), and (ix) resource deallocation.

In the proposed model, the queue is modeled by priorities. The moment resources are available, the priority patient is evaluated by the intensivist. The patient's hospitalization admission or refusal depends on prognosis and potential benefit generated by therapeutic interventions. The admission criteria and discharge from the intensive care unit are established 
according to the ICU Admission, Discharge, and Triage Guidelines, accepted by the Society of Critical Care Medicine ${ }^{57}$. Thus, patients referred for ICU admission are prioritized according to their condition, as expressed in Table 1.

\begin{tabular}{|c|c|c|c|c|}
\hline $\begin{array}{c}\text { admission } \\
\text { priority }\end{array}$ & $\begin{array}{c}\text { recovery } \\
\text { probability }\end{array}$ & $\begin{array}{c}\text { therapeutic support } \\
\text { limitation }\end{array}$ & $\begin{array}{c}\text { need for } \\
\text { intervention }\end{array}$ & $\begin{array}{c}\text { need for } \\
\text { monitoring }\end{array}$ \\
\hline 1 & high & no & yes & yes \\
\hline 2 & high & no & no & yes \\
\hline 3 & low & yes & yes & yes \\
\hline 4 & low & yes & no & yes \\
\hline 5 & null & yes & yes & yes \\
\hline
\end{tabular}

Table 1. Patient admission priority classification.

Patients classified as priority 2 and priority 4 according to Table 1 have a high risk of needing immediate intervention, so they should be monitored. Patients assessed as priority 5 are usually terminally ill, dying or potential organ donors, indicated to be in ICU only when there is a medical specification.

The intensive care unit is an open system in which patients can arrive at any time. Thus, the number of patients in queue and resource usage vary over time. Here, we propose ICU performance metric such as the relationship between the number of admissions $n_{a}$, length of stay $l_{s}$, number of patients in queue $n_{q}$, and number of beds in use $n_{b}$ for a given period of time. The ICU performance metric expression is given by:

$$
\eta_{i c u}=\frac{\frac{n_{a}}{n_{a_{r e f}}}+\frac{l_{s_{r e f}}-l_{s}}{l_{s_{r e f}}}+\frac{n_{q_{r e f}}-n_{q}}{n_{q_{r e f}}}+\frac{n_{b}}{n_{b_{r e f}}}}{4}
$$

where the variables $n_{a_{r e f}}, l_{s_{r e f}}, n_{q_{r e f}}$ and $n_{b_{r e f}}$ are fixed at the maximum reference value of variables $n_{a}, l_{s}, n_{q}$ e $n_{b}$, respectively.

The complexity measure is calculated using (3) during simulation of the model shown in Figure 4. Each patient in queue adds a connection to the system and when hospitalized the patient adds three to four connections. After admission, the patient connects with the bed, the equipment, the staff, and the adverse events (if affected by any adverse event).

\section{Results}

The intensive care unit proposed model represents connections between system elements. Patients in queue establish a connection with the patient in front of them or with the intensivist physician at the time of admission. When admitted, the patient establishes a connection with the bed, equipment, staff, and adverse events (if they occur). Initially, we defined the optimized system configuration case: an adequate resources number to meet demand and adverse events rate equal to non-preventable events rate.

In the optimized case, patient arrival rate represented a normal distribution with 36 hours mean and 4 hours standard deviation, $N(36,4)$. In other words, every 36 hours on average there is one ICU bed request. Patients were ranked by priority, as follows: $35 \%$ for Priority 1, 50\% for Priority 2, 7\% for Priority 3, 7\% for Priority 4, and $1 \%$ for Priority 5. The waiting queue type was first in, first out (FIFO), conditioned to priority ordering. As for resources, the ICU configuration consisted of 10 beds, $100 \%$ of equipment, and $100 \%$ of the staff workload, with a 5\% addition margin. This margin refers to cases in which the professional needs to extend their working hours, for example, cardiac resuscitation close to the staff's shift change. The admission refusal rate was set at $10 \%$. For each admitted patient, the following were allocated: one bed, from $6 \%$ to $12 \%$ of the total equipment and from $6 \%$ to $12 \%$ of the staff's workload, following a uniform distribution $U(6,12)$ for equipment and staff. The ICU average length of stay, in days, was related to the patient's priority ${ }^{58}$, represented by a normal distribution equal to: $N(8,3)$ for Priority $1, N(5,2)$ for Priority $2, N(7,1)$ for Priority $3, N(7,1)$ for Priority 4 and $N(30,7)$ for Priority 5.

Based on the medical literature ${ }^{13-15}$, the adverse event rate was set at $12 \%$. For each adverse event occurrence, patient length of stay was increased by a period between 15 and 45 days, represented by a $U(15,45)$ uniform distribution. The ICU death rate was set at $20 \%$. The patient leaves when he or she is discharged or dies, situations in which it's reserved resources are deallocated. For cases of admission refusal, the patient leaves the system before entering the ICU room. Patients who received intensive care can be classified as (i) recovered with no adverse event history, (ii) deceased with no adverse event history, (iii) recovered with adverse event history, and (iv) deceased with adverse event history. 
The System performance was measured using the expression (4) represented metric, which relates the admissions number $n_{a}$, length of stay $l_{s}$, patients in queue $n_{q}$ and beds in use $n_{b}$. The values of the variables $n_{a_{r e f}}, l_{s_{r e f}}, n_{q_{r e f}}$ and $n_{b_{r e f}}$ present in the expression (4) were determined through experiments, considering the maximum value that each variable can assume.

To determine the variables $n_{a}, l_{s}, n_{q}$ and $n_{b}$ maximum values, the length of stay distribution representing all patients is first calculated. Mean and standard deviation values were weighted by the frequency of each patient's priority. The result obtained was equal to 6.58 days in ICU on average with an 2.26 days average standard deviation. Thus, the maximum ICU stay was estimated as the average (6.58 days) plus three deviations, that is, approximately 14 days. As $12 \%$ of patients have adverse events and their time in the ICU increases on average 30 days, the maximum time was recalculated, considering that $88 \%$ of total patients would be hospitalized for 14 days and $12 \%$ of patients would stay in the ICU for about 44 days, resulting in a maximum time of 17.6 days.

Then, the maximum queue size was calculated, considering critical arrival rates: $N(4,4), N(6,4)$ and $N(12,4)$. The average of these arrival rates means resulted in 7.33 hours, which would correspond to an average of 3 patients arriving per day and 1194 patients per year (simulated period) approximately. If a minimum number of patients were treated during the year, that is, when each patient length of stay was maximum (17.6 days), the total number of patients who would go into an ICU with 10 beds would be 207. Thus, the maximum queue size would be 990 patients approximately.

The maximum admitted patients was calculated based on the average length of stay distribution $N(6.58,2.26)$. Considering adverse events rate, we consider that $88 \%$ of the total number of patients admitted stays in ICU for 6.58 days and $12 \%$ stays for 36.58 days, on average. Therefore, the average length of stay is 10.18 days. In one year, 360 patients, approximately, are admitted into an ICU with 10 beds.

We define the reference for the maximum number of beds in use as $85 \%$ of the total beds. Thus, the bed availability is kept within the expected limits for the ICU, according to McManus ${ }^{16}$. Since the modeled ICU has 10 beds, whenever the mean number of beds in use exceeds 8.5, the expression (5) determines the fourth installment numerator of performance metric. This modeling is a way to penalize situations in which the ICU is working at its limit.

$$
\begin{cases}n_{b} & \text { if } \quad n_{b}<8.5 \\ n_{b_{r e f}}-5 \cdot\left(n_{b}-n_{b_{r e f}}\right) & \text { if } \quad n_{b} \geq 8.5\end{cases}
$$

Table 2 summarizes the calculated values for the variables $n_{a_{r e f}}, l_{s_{r e f}}, n_{q_{r e f}}$ and $n_{b_{r e f}}$, which represent maximum reference values for the variables $n_{a}, l_{s}, n_{q}$ and $n_{b}$, respectively.

\begin{tabular}{|c|c|l|}
\hline variable & value & description (maximum mean value of...) \\
\hline$n_{a_{r e f}}$ & 360 & ...admissions number \\
\hline$l_{s_{\text {ref }}}$ & 17.6 & ...length of stay \\
\hline$n_{q_{r e f}}$ & 990 & ...number of patients in queue \\
\hline$n_{b_{r e f}}$ & 8.5 & ...number of beds in use \\
\hline
\end{tabular}

Table 2. Reference values for variables $n_{a}, l_{s}, n_{q}$ and $n_{b}$.

After defining probabilistic distributions and reference values for calculating system performance, we performed the ICU parameters sensitivity study: number of beds $n_{\text {bed }}$, percentage of equipment $n_{\text {equipment }}$, percentage of staff $n_{\text {staff }}$, adverse event rate $r_{a e}$ and arrival rate mean $\mu_{\text {arrival }}$ (relative to the normal distribution with standard deviation equal to 4 hours).

\section{Case study 1: ICU model sensitivity analysis}

The ICU model sensitivity analysis was developed using the area method. This method defines sensitivity index value of each parameter as the relation between the parameter variation area and total area. The areas comprise the surface between the curves and base axis, parallel line to abscissa axis, which presents a constant value in the ordinate corresponding to the system output referring to the base case $\vec{\beta}$.

The base value for number of beds $n_{\text {bed }}$ is 10 , percentage of equipment $n_{\text {equipment }}$ is $100 \%$, percentage of staff $n_{\text {staff }}$ is $105 \%$, adverse event rate $r_{a e}$ is $12 \%$ and arrival rate mean $\mu_{\text {arrival }}$ is $36 h$. These values were defined empirically, considering the desired operating conditions of the ICU (optimized base case). The scenarios are built modifying the parameters $n_{b e d}$ (from 1 bed to 20 beds), $n_{\text {equipment }}$ (from $15 \%$ to $200 \%$ ), $n_{\text {staff }}$ (from $15 \%$ to $200 \%$ ), $r_{a e}$ (from $12 \%$ to $24 \%$ ) and $\mu_{\text {arrival }}$ (from $4 h$ to $72 h)$.

We simulate the ICU operation for a one year period. Considering the ICU optimized configuration (optimized base case), an average of 193.79 patients were admitted to the ICU, who stayed hospitalized for about 8.49 days. The queue mean size was 
5.69 patients with approximately 8.67 beds utilization during the simulated period. The mean performance calculated using the expression (4) was $74 \%$.

Figure 6 refers to the base case spider diagram, in which the ICU working condition is regular. In Figure 6, the number of beds $n_{b e d}$ negative variation (in blue) generated the greater impact on performance. The parameters percentage of equipment $n_{\text {equipment }}$ (in orange) and staff percentage $n_{\text {staff }}$ (in yellow) showed similar behavior, with a significant impact for values below the base value. The adverse event rate $r_{a e}$ (in purple) was only varied from $0 \%$ to $100 \%$, since the estimated base value refers to non-preventable events. The base value $r_{a e}$ was equal to the smallest feasible value for the model in question. The increase in adverse event rate by $100 \%$ led to a drop in performance greater than $10 \%$. The arrival rate parameter $\mu_{\text {arrival }}$ mean variation (in green) led to lower performance values in both contexts analysis (negative variation and positive variation).

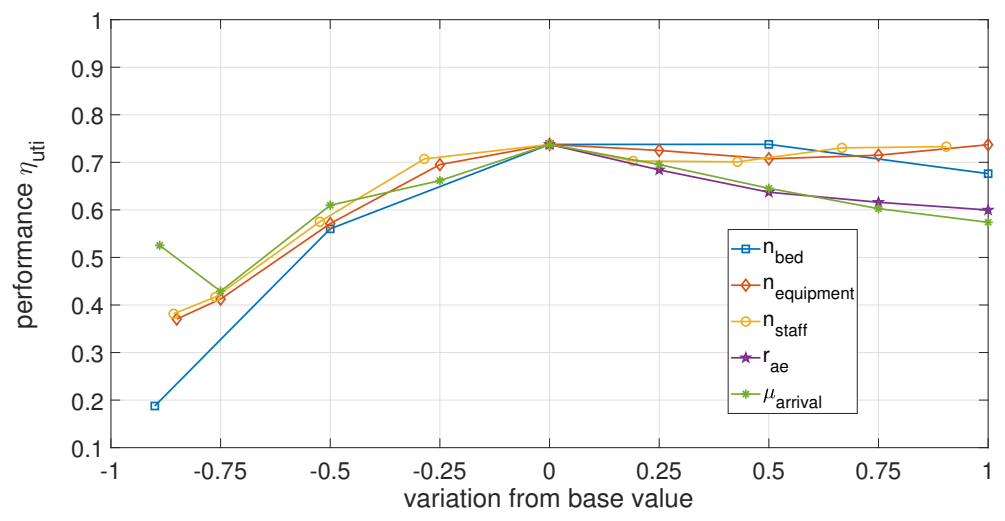

Figure 6. Spider-diagram of the ICU system for optimized base case.

From one-at-a-time measures, visually presented in the spider diagram in Figure 6, the area method was applied to calculate the sensitivity indices. The values obtained were: (i) $S_{n_{b e d}}^{a}=0.2556$, (ii) $S_{n_{\text {equipment }}}^{a}=0.1904$, (iii) $S_{n_{\text {staff }}}^{a}=0.1757$, (iv) $S_{r_{a e}}^{a}=0.1121$, and (v) $S_{\mu_{\text {arrival }}}^{a}=0.2662$. The most sensitive parameter was $\mu_{\text {arrival }}$, followed by the $n_{\text {bed }}$. The sensitivity index values are used to define system connections relevance values. In this way, after completing the sensitivity analysis step, we can calculate the ICU system complexity.

\section{Case study 2: ICU system complexity calculation}

The complexity metric application using the expression (3) depends on the probability $P(c)$ definition and the relevance $\gamma_{c}$ of the system connections. The probabilities of each connection type in the ICU system are presented in Table 3 . The queue connection probabilities were defined experimentally considering each patient priority. The experimental probability refers to the number of patients per priority in the queue at each analyzed instant in regards to the total number of patients in the queue. Probability values for resources and adverse events were defined according to the base case configuration.

\begin{tabular}{|c|c|}
\hline connection $c$ & $P(c)$ \\
\hline patient priority 1 - queue & 0.06 \\
\hline patient priority 2 - queue & 0.40 \\
\hline patient priority 3 - queue & 0.19 \\
\hline patient priority 4 - queue & 0.29 \\
\hline patient priority 5 - queue & 0.06 \\
\hline patient - bed & 0.10 \\
\hline patient - equipment & 0.09 \\
\hline patient - staff & 0.09 \\
\hline patient - adverse event & 0.12 \\
\hline
\end{tabular}

Table 3. Occurrence probabilities of connections in ICU system.

With 10 beds in the proposed configuration, the patient connection probability to the bed is 0.1 . Thus, each patient requires an average of 0.09 of the total equipment and the total team workload. This value 0.09 refers to the uniform distribution 
$U(6,12)$ mathematical expectation, used to define the equipment or staff quantity required by each patient. As for adverse events, the connection probability refers to it's rate, that is 0.12 .

The system connections relevance $\gamma_{c}$ was defined based on the parameters sensitivity indices. We check which parameters directly influence each connection and thus define the relevance value $\gamma_{c}$, as shown in Table 4 .

\begin{tabular}{|c|c|}
\hline connection $c$ & $\gamma_{c}$ \\
\hline patient - queue & $S_{\mu_{\text {arrival }}}^{a}$ \\
\hline patient - bed & $S_{n_{\text {bed }}}^{a}$ \\
\hline patient - equipment & $S_{n_{\text {equipment }}}^{a}$ \\
\hline patient - staff & $S_{n_{\text {staff }}}^{a}$ \\
\hline patient - adverse event & $S_{r_{\text {ae }}}^{a}$ \\
\hline
\end{tabular}

Table 4. Connections relevance of the ICU system.

The complexity value was calculated from the system dynamics, using the expression (3). The optimized base case complexity was $\psi^{i c u}(c, \gamma)=20.30$ (considering the mean obtained after 100 system simulation repetitions for a one year period). The value of $\psi^{i c u}(c, \gamma)$ consists of the active connections evaluation during ICU operation, which are weighted by their relevance. As the relevance $\gamma_{c}$ of each connection is given by sensitivity indices, the metric $\psi^{i c u}(c, \gamma)$ considers the external environment influence on the system performance, observing the relationship between the parts and the whole.

The obtained complexity value ( $\left.\psi^{i c u}(c, \gamma)=20.30\right)$ can be understood when compared to complexity values from other scenarios. We modified the arrival rate mean $\mu_{\text {arrival }}$ to analyze overload cases, as shown in Table 5 . The impact of changing the arrival rate was verified by simulating the scenarios $\alpha^{12 h}$ and $\alpha^{24 h}$, since the number of resources and adverse events rate were kept equal to the optimized base case $\alpha^{36 h}$. The variables that make up the ICU performance measure, expressed by (4), were obtained after simulating each scenario and their values are shown in Table 6.

\begin{tabular}{|c|c|c|c|c|c|c|}
\hline base case & condition & $n_{\text {bed }}$ & $n_{\text {equipment }}$ & $n_{\text {staff }}$ & $r_{\text {ae }}$ & $\mu_{\text {arrival }}$ \\
\hline$\alpha^{12 h}$ & overload & 10 & $100 \%$ & $105 \%$ & $12 \%$ & $12 h$ \\
\hline$\alpha^{24 h}$ & overload & 10 & $100 \%$ & $105 \%$ & $12 \%$ & $24 h$ \\
\hline$\alpha^{36 h}$ & regularity & 10 & $100 \%$ & $105 \%$ & $12 \%$ & $36 h$ \\
\hline
\end{tabular}

Table 5. ICU system overload and regularity cases.

\begin{tabular}{|c|c|c|c|c|c|}
\hline base case & $\begin{array}{c}\text { admissions } \\
n_{a}\end{array}$ & $\begin{array}{c}\text { length of stay } \\
l_{s} \text { [days] }\end{array}$ & $\begin{array}{c}\text { patients in queue } \\
n_{q}\end{array}$ & $\begin{array}{c}\text { beds in use } \\
n_{b}\end{array}$ & $\begin{array}{c}\text { performance } \\
\eta_{\text {icu }}\end{array}$ \\
\hline$\alpha^{12 h}$ & 179.64 & 9.75 & 244.09 & 9.31 & 0.56 \\
\hline$\alpha^{24 h}$ & 194.80 & 8.98 & 58.44 & 9.22 & 0.64 \\
\hline$\alpha^{36 h}$ & 193.79 & 8.49 & 5.69 & 8.67 & 0.74 \\
\hline
\end{tabular}

Table 6. Simulation data of ICU performance components variables for overload and regularity cases.

Under overload condition, the performance values obtained were inferior to the optimized base case performance: $56 \%$ relative to $\alpha^{12 h}$ and 64\% relative to $\alpha^{24 h}$. The Table 6 data indicate a queue size $n_{q}$ increase by more than $1000 \%$ and ICU occupation above the recommended value (85\%), since the ICU had $93.1 \%$ beds in use for the case $\alpha^{12 h}$ and $92.2 \%$ for the case $\alpha^{24 h}$. The average number of patients in the queue according to each priority is shown in Figure 7 and in Table 7. As the available resources were insufficient to meet the demand, the number of patients in the queue increased significantly (and in a non-linear manner) with the arrival rate mean reduction.

Based on Figure 7 and Table 7, we verified that the largest number of patients in the queue refers to priority 2 patients. However, regarding the regularity case, these patients represented $38.01 \%$ of the total patients in the queue, while in overload cases, priority 2 patients represented more than $60 \%$ of the total patients in the queue. The lowest values in Table 7 are priority 1 and priority 5 patients. Most of the priority 1 patients occupied ICU beds while priority 5 patients were the smallest group with ICU beds demand. Both priority 3 and priority 4 patients percentage increased as the arrival rate mean also increased. 


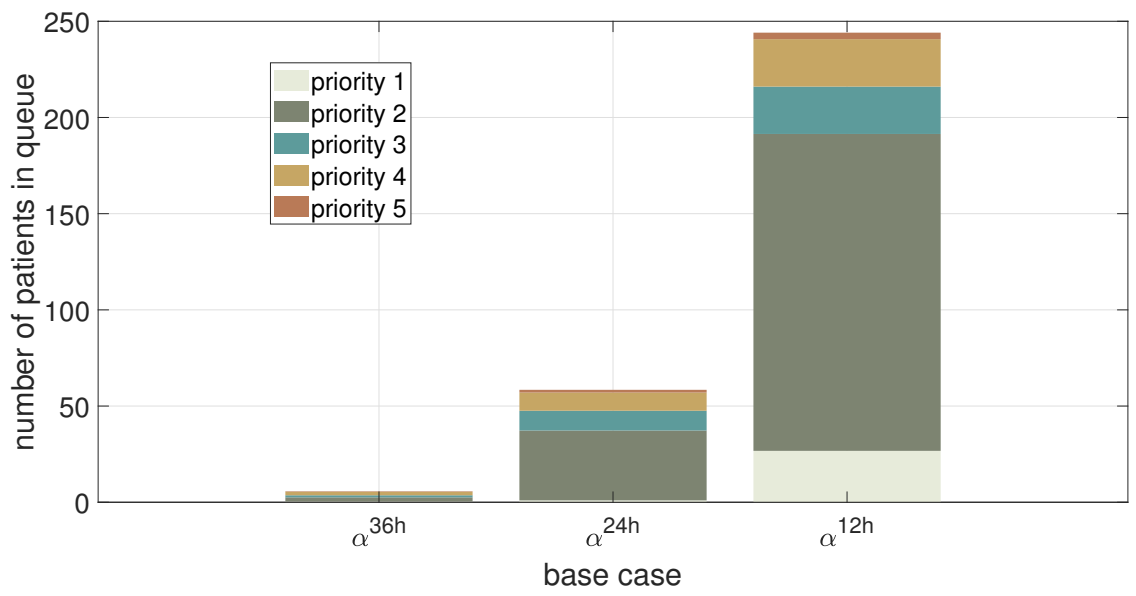

Figure 7. Number of patients per priority in the ICU queue for base cases $\alpha^{36 h}, \alpha^{24 h}$ and $\alpha^{12 h}$.

\begin{tabular}{|c|c|c|c|c|c|c|}
\hline \multirow{2}{*}{$\begin{array}{c}\text { patient } \\
\text { priority }\end{array}$} & \multicolumn{3}{|c|}{ number of patients in queue } & \multicolumn{3}{c|}{ percentage of patients in queue } \\
\cline { 2 - 6 } & $\alpha^{\mathbf{1 2 h}}$ & $\alpha^{\mathbf{2 4 h}}$ & $\alpha^{\mathbf{3 6 h}}$ & $\alpha^{\mathbf{1 2 h}}$ & $\alpha^{\mathbf{2 4 h}}$ & $\alpha^{\mathbf{3 6 h}}$ \\
\hline 1 & 26.71 & 0.97 & 0.38 & $10.94 \%$ & $1.68 \%$ & $6.83 \%$ \\
\hline 2 & 164.72 & 36.30 & 2.16 & $67.48 \%$ & $62.13 \%$ & $38.01 \%$ \\
\hline 3 & 24.62 & 10.28 & 1.07 & $10.09 \%$ & $17.61 \%$ & $18.95 \%$ \\
\hline 4 & 24.66 & 9.44 & 1.80 & $10.10 \%$ & $16.16 \%$ & $31.77 \%$ \\
\hline 5 & 3.36 & 1.42 & 0.25 & $1.39 \%$ & $2.42 \%$ & $4.44 \%$ \\
\hline
\end{tabular}

Table 7. Number/Percentage of patients by priority in ICU queue relative to overload and regularity cases.

The calculated complexity values for overload scenarios $\alpha^{12 h}$ and $\alpha^{24 h}$ were 153.42 and 62.34 , respectively. The complexity of these scenarios was 3 to $7.5 \times$ greater than the regularity scenario $\alpha^{36 h}$ complexity. The increase overload cases complexity is due to the patient increase in the system. These patients may be waiting for bed in the admission queue (due to resources unavailability) or inside the unit (generating a high resource utilization rate).

\section{Discussion}

The intensive care unit is a complex system composed of several connected and interdependent elements. Thus, when adverse events occur, the entire system is affected. Prolonged hospitalizations are a stressful situation for the patient (and their families) and for the staff. When these circumstances occur, part of the resources are allocated for a long period, which affects the patients admission. Considering the ICU system relevance for health recovery, the system as a whole must show reliability and robustness.

The ICU system shows robust behavior when: (i) the adverse events occurrence is reduced, (ii) the patients flow occurs regularly, and (iii) the proper functioning is maintained even in instability situations, such as unexpected demand increase, lack of human resources or equipment failure. To promote ICU system robustness, redundancy mechanisms can be adopted, such as functional overlap between health professionals. In certain situations, these professionals may perform specific procedures in common or use multifunctional critical care apparatus ${ }^{59-61}$. The redundancy implementation in the ICU allows an adequate response to small disturbances and to the some of its components failure.

However, increasing ICU system elements to obtain redundancy increases its complexity level. Overload situations also lead to increased complexity, as we've seen when simulating different patient arrivals rates. Thus, both the redundancy mechanisms implementation and the ICU system exposure to different demand regimes can impact the value obtained for its complexity $\psi^{i c u}(c, \gamma)$.

We can investigate aspects related to ICU system robustness by observing the complexity variation. System changes, such as idleness or overload, can affect the parameters sensitivity and the connections entropy. The robustness analysis, associated with the specialists expertise in the critical care area, contributes to decision-making in the ICU systems management. These decisions can improve the resources allocation and adjust the system's operating rules. 
Overload situations in ICU systems can occur both during their routine operation and in exceptional situations, such as the current COVID-19 pandemic. Individuals contaminated with new coronavirus or SARS-CoV-2 can present symptoms with varying degrees of severity, from asymptomatic to serious cases, in which the patient develops acute respiratory syndrome accompanied or not by other vital systems impairment. In severe cases, the patient's admission to the ICU is recommended ${ }^{62,63}$.

The high number of serious COVID-19 cases recorded in several countries in recent months has caused a situation of generalized overload in health systems at a global level, especially those aimed at intensive care ${ }^{64}$. ICU occupation rates above $85 \%$ - reaching $100 \%$ in numerous cases - have been observed since the pandemic beginning ${ }^{65}$. The ICU systems overload is addressed in studies performed in hospitals in China ${ }^{66}$ and the United States, the main pandemic epicenters ${ }^{67,68}$, in Italy, one most affected countries by the pandemic in contaminated percentage $e^{69,70}$, in the Netherlands, Germany ${ }^{70}$ and around 216 countries analyzed by Rocks and Idriss $(2020)^{71}$ and Sen-Crowe et al. $(2021)^{64}$.

From the hierarchical organization of complex systems point of view, the ICU system has subsystems meanwhile is part of a larger system: the hospital system. The ICU complexity increase can affect the entire hospital system complexity. The proposed complexity metric can be used to evaluate the subsystem complexity relationship with the system that comprises it. Regarding the internal dynamics, the proposed metric can contribute to the complexity evaluation when there are system changes, such as lack of beds, lack or failure of equipment and lack of professionals. Future studies can also use the proposed metric to measure the effect (in terms of complexity) of increasing demand for ICU beds, resulting from the growing number of COVID-19 serious cases.

\section{Conclusion}

This work proposed a system complexity metric based on weighted connections. We use sensitivity indices to weight the connections between system components. Thus, the influence of the external environment could be verified through the sensitivity analysis. The simulation of the intensive care unit system provided us: (i) average number of patients admitted, (ii) average ICU length of stay, (iii) number of patients by priority in the queue, and (iv) system resources usage. Using the simulation data, we calculate the system performance, the ICU system's parameters sensitivity and complexity, under regularity and overload conditions. The intensive care unit presents complex system characteristics, as the interaction between its components generates functionality (critical care) that is impossible to obtain by its parts separately. In addition, the ICU system has the self-organizing characteristic, observed when professionals need to extend their working hours or when there is a need for overlapping functional competencies. The hierarchical organization is also observable, given that the system's functioning and structures can be analyzed at different levels. Based on the results obtained in this work, we list some future investigations: (i) sensitivity analysis and complexity study of idleness cases and system overload in comparison with the regularity case, (ii) analysis regarding performance measure relations and system complexity, and (iii) metrics development to define regions of system robustness.

\section{Data Availability}

Some or all data, models, or code that support the findings of this study are available from the corresponding author upon request.

\section{References}

1. Page, S. E. Diversity and complexity (Princeton University Press, New Jersey, USA, 2010).

2. Kannampallil, T. G., Schauer, G. F., Cohen, T. \& Patel, V. L. Considering complexity in healthcare systems. J. biomedical informatics 44, 943-947, DOI: https://doi.org/10.1016/j.jbi.2011.06.006 (2011).

3. Holland, J. H. Complexity: A very short introduction (Oxford University Press, Great Clarendon Street, Oxford, United Kingdom, 2014), 1 edn.

4. Morin, E. Introducción al pensamiento complejo [Introduction to complex thinking] (Gedisa, Barcelona, 2001), 5 edn.

5. Deacon, T. \& Koutroufinis, S. Complexity and dynamical depth. Information 5, 404-423, DOI: https://doi.org/10.3390/ info5030404 (2014).

6. Kurths, J. et al. General remarks on complexity. In Inside Versus Outside, vol. 63, 219-234, DOI: https://doi.org/10.1007/ 978-3-642-48647-0_13 (Springer, Berlin, Heidelberg, 1994).

7. Mainzer, K. \& Chua, L. The universe as automaton: From simplicity and symmetry to complexity (Springer Science \& Business Media, Berkeley, California, USA, 2011), 1 edn.

8. Mitchell, M. Complexity: A guided tour (Oxford University Press, Oxford, United Kingdom 2009, 2009 ), 1 edn. 
9. Bak, P. How nature works: the science of self-organized criticality (Copernicus Books, New York, NY, USA, 1996), 1 edn.

10. Simon, H. A. The architecture of complexity. Proc. Am. Philos. Soc. 106, 467-482, DOI: https://doi.org/10.1007/ 978-1-4899-0718-9_31 (1962).

11. Bar-Yam, Y. General features of complex systems. Encycl. Life Support. Syst. (EOLSS), UNESCO 1, 1-10 (2002).

12. Mobus, G. E. \& Kalton, M. C. Principles of systems science (Springer, New York, NY, USA, 2015), 1 edn.

13. Rafter, N. et al. Adverse events in healthcare: learning from mistakes. QJM: An Int. J. Medicine 108, 273-277, DOI: https://doi.org/10.1093/qjmed/hcu145 (2014).

14. Rothschild, J. M. et al. The critical care safety study: The incidence and nature of adverse events and serious medical errors in intensive care. Critical care medicine 33, 1694-1700, DOI: https://doi.org/10.1097/01.ccm.0000171609.91035.bd (2005).

15. Forster, A. J., Kyeremanteng, K., Hooper, J., Shojania, K. G. \& van Walraven, C. The impact of adverse events in the intensive care unit on hospital mortality and length of stay. BMC health services research 8, 259, DOI: https: //doi.org/10.1186/1472-6963-8-259 (2008).

16. McManus, M. L., Long, M. C., Cooper, A., Litvak, E. et al. Queuing theory accurately models the need for critical care resources. Anesthesiology 100, 1271-1276, DOI: https://doi.org/10.1186/1472-6963-8-259 (2004).

17. Cardoso, L. T. et al. Impact of delayed admission to intensive care units on mortality of critically ill patients: a cohort study. Critical care 15, R28, DOI: https://doi.org/10.1186/cc9975 (2011).

18. Town, J. A. et al. Relationship between icu bed availability, icu readmission, and cardiac arrest on the general wards. Critical care medicine 42, 2037, DOI: https://doi.org/10.1097/CCM.0000000000000401 (2014).

19. Guazzini, A. et al. Humans best judge how much to cooperate when facing hard problems in large groups. Sci. reports $\mathbf{9}$, 5497, DOI: https://doi.org/10.1038/s41598-019-41773-2 (2019).

20. Carayon, P. et al. Characterising the complexity of medication safety using a human factors approach: an observational study in two intensive care units. BMJ Qual Saf 23, 56-65, DOI: https://doi.org/10.1136/bmjqs-2013-001828 (2014).

21. Bricon-Souf, N., Renard, J.-M. \& Beuscart, R. Dynamic workflow model for complex activity in intensive care unit. Int. journal medical informatics 53, 143-150, DOI: https://doi.org/10.1016/S1386-5056(98)00155-5 (1999).

22. Bertalanffy, L. v. General system theory: Foundations, development, applications (Braziller. New York, 1968).

23. Maier, M. W. Architecting principles for systems-of-systems. In INCOSE International Symposium, vol. 6, 565-573, DOI: https://doi.org/10.1002/j.2334-5837.1996.tb02054.x (Wiley Online Library, 1996).

24. Rechtin, E. \& Maier, M. W. The art of systems architecting (CRC Press, 2010).

25. Klir, G. J. Facets of systems science, vol. 7 (Springer Science \& Business Media, 2013).

26. Wilson, M. P. General system theory: Towards the unification of science. In Proceedings of the 58th Annual Meeting of the ISSS-2014 United States, vol. 1 (2015).

27. Tarantola, S., Gatelli, D., Kucherenko, S., Mauntz, W. et al. Estimating the approximation error when fixing unessential factors in global sensitivity analysis. Reliab. Eng. \& Syst. Saf. 92, 957-960, DOI: https://doi.org/10.1016/j.ress.2006.07.001 (2007).

28. Cassandras, C. G. \& Lafortune, S. Introduction to discrete event systems (Springer Science \& Business Media, 2009), 2 edn.

29. Wainer, G. A. Discrete-event modeling and simulation: a practitioner's approach (CRC press, Southern Gate, Chichester, UK, 2009), 1 edn.

30. Pegden, C. D., Sadowski, R. P. \& Shannon, R. E. Introduction to simulation using SIMAN (McGraw-Hill, Inc., 1995).

31. Holland, J. H. Studying complex adaptive systems. J. Syst. Sci. Complex. 19, 1-8, DOI: https://doi.org/10.1007/ s11424-006-0001-z (2006).

32. Simon, H. A. How complex are complex systems? In PSA: Proceedings of the biennial meeting of the Philosophy of Science Association, 507-522, DOI: https://doi.org/10.1086/psaprocbienmeetp.1976.2.192399 (JSTOR, 1976).

33. Casti, J. L. Complexity and simplicity, in the eye of the beholder. Complexity 1, 2-3, DOI: https://doi.org/10.1002/cplx. 6130010202 (1995).

34. Bar-Yam, Y. Dynamics of complex systems, vol. 213 (Addison-Wesley, Newton, Massachusetts, USA, 1997), 1 edn. 
35. Gell-Mann, M. Simplicity and complexity in the description of nature. Eng. Sci. 51, 2-9 (1988).

36. Gershenson, C. \& Fernández, N. Complexity and information: Measuring emergence, self-organization, and homeostasis at multiple scales. Complexity 18, 29-44, DOI: https://doi.org/10.1002/cplx.21424 (2012).

37. Gell-Mann, M. The Quark and the Jaguar: Adventures in the Simple and the Complex (Macmillan, 1995).

38. Lloyd, S. Measures of complexity: a nonexhaustive list. IEEE Control. Syst. Mag. 21, 7-8, DOI: https://doi.org/10.1109/ MCS.2001.939938 (2001).

39. Shannon, C. E. A mathematical theory of communication. The Bell Syst. Tech. J. 27, 379-423, 623-656, DOI: https: //doi.org/10.1002/j.1538-7305.1948.tb00917.x (1948).

40. Kolmogorov, A. N. Three approaches to the quantitative definition of information. Probl. information transmission 1, 1-7 (1965).

41. Zurek, W. H. Thermodynamic cost of computation, algorithmic complexity and the information metric. Nature 341, 119, DOI: https://doi.org/10.1038/341119a0 (1989).

42. Wolfram, S. Universality and complexity in cellular automata. Phys. D: Nonlinear Phenom. 10, 1-35, DOI: https: //doi.org/10.1016/0167-2789(84)90245-8 (1984).

43. Lloyd, S. \& Pagels, H. Complexity as thermodynamic depth. Annals physics 188, 186-213, DOI: https://doi.org/10.1016/ 0003-4916(88)90094-2 (1988).

44. Crutchfield, J. P. \& Young, K. Inferring statistical complexity. Phys. Rev. Lett. 63, 105, DOI: https://doi.org/10.1103/ PhysRevLett.63.105 (1989).

45. Theiler, J. Estimating fractal dimension. JOSA A 7, 1055-1073, DOI: https://doi.org/10.1364/JOSAA.7.001055 (1990).

46. Bennett, C. H. Logical depth and physical complexity. The Univers. Turing Mach. A Half-Century Surv. 207-235, DOI: https://doi.org/10.1007/978-3-7091-6597-3_8 (1995).

47. Paiva, J. et al. Metric for calculation of system complexity based on its connections. Transactions on Environ. Electr. Eng. 2, 67-73, DOI: https://doi.org/10.22149/teee.v2i1.80 (2017).

48. Critchfield, G. C., Willard, K. E. \& Connelly, D. P. Probabilistic sensitivity analysis methods for general decision models. Comput. biomedical research 19, 254-265, DOI: https://doi.org/10.1016/0010-4809(86)90020-0 (1986).

49. Homma, T. \& Saltelli, A. Importance measures in global sensitivity analysis of nonlinear models. Reliab. Eng. \& Syst. Saf. 52, 1-17, DOI: https://doi.org/10.1016/0951-8320(96)00002-6 (1996).

50. Saltelli, A., Tarantola, S., Campolongo, F. \& Ratto, M. Sensitivity analysis in practice: a guide to assessing scientific models (John Wiley \& Sons, 2004).

51. Pannell, D. J. Sensitivity analysis: strategies, methods, concepts, examples. Agric Econ 16, 139-152 (1997).

52. Frey, C. \& Patil, S. R. Identification and review of sensitivity analysis methods. Risk analysis 22, 553-578, DOI: https://doi.org/10.1111/0272-4332.00039 (2002).

53. Gomes, V. M. Complexidade Natural de Sistemas Baseada em Análise de Sensibilidade. Ph.D. thesis, Universidade Federal de Goiás (2020).

54. Hamby, D. A comparison of sensitivity analysis techniques. Heal. physics 68, 195-204, DOI: https://doi.org/10.1097/ 00004032-199502000-00005 (1995).

55. Eschenbach, T. G. \& McKeague, L. S. Exposition on using graphs for sensitivity analysis. The Eng. Econ. 34, 315-333, DOI: https://doi.org/10.1080/00137918908902996 (1989).

56. Gomes, V. M., Paiva, J. R., Reis, M. R., Wainer, G. A. \& Calixto, W. P. Mechanism for measuring system complexity applying sensitivity analysis. Complexity 2019, DOI: https://doi.org/10.1155/2019/1303241 (2019).

57. Nates, J. L. et al. Icu admission, discharge, and triage guidelines: a framework to enhance clinical operations, development of institutional policies, and further research. Critical care medicine 44, 1553-1602, DOI: https://doi.org/10.1097/CCM. 0000000000001856 (2016).

58. Caldeira, V. M. H. et al. Criteria for patient admission in the intensive care unit and mortality rates. Braz. Med. Assoc. J. 56, 528-34, DOI: https://doi.org/10.1590/S0104-42302010000500012 (2010).

59. Grogan, E. L. et al. The impact of aviation-based teamwork training on the attitudes of health-care professionals. $J$. Am. Coll. Surg. 199, 843-848, DOI: https://doi.org/10.1016/j.jamcollsurg.2004.08.021 (2004). 
60. Leming-Lee, S. et al. Crew resource management in perioperative services: navigating the implementation road map. $J$. clinical outcomes management: JCOM 12, 353-8 (2005).

61. Véliz, P. L., Berra, E. M. \& Jorna, A. R. Definition of specific functions and procedural skills required by cuban specialists in intensive care and emergency medicine. Medicc Rev. 17, 18-26 (2015).

62. Chen, N. et al. Epidemiological and clinical characteristics of 99 cases of 2019 novel coronavirus pneumonia in wuhan, china: a descriptive study. The lancet 395, 507-513, DOI: https://doi.org/10.1016/S0140-6736(20)30211-7 (2020).

63. Huang, C. et al. Clinical features of patients infected with 2019 novel coronavirus in wuhan, china. The lancet 395 , 497-506, DOI: https://doi.org/10.1016/S0140-6736(20)30183-5 (2020).

64. Sen-Crowe, B., Sutherland, M., McKenney, M. \& Elkbuli, A. A closer look into global hospital beds capacity and resource shortages during the covid-19 pandemic. J. Surg. Res. 260, 56-63, DOI: https://doi.org/10.1016/j.jss.2020.11.062 (2020).

65. Organization, W. H. Coronavirus disease (covid-19) pandemic (2021). https://www.who.int/emergencies/diseases/ novel-coronavirus-2019 Last accessed on 2021-09-03.

66. Shang, Y. et al. Management of critically ill patients with covid-19 in icu: statement from front-line intensive care experts in wuhan, china. Annals intensive care 10, 1-24, DOI: https://doi.org/10.1186/s13613-020-00689-1 (2020).

67. Bravata, D. M. et al. Association of intensive care unit patient load and demand with mortality rates in us department of veterans affairs hospitals during the covid-19 pandemic. JAMA network open 4, e2034266-e2034266, DOI: https: //doi.org/10.1001/jamanetworkopen.2020.34266 (2021).

68. Janke, A. et al. Analysis of hospital resource availability and covid-19 mortality across the united states. J. Hosp. Medicine 16, 211-214, DOI: https://doi.org/10.12788/jhm.3539 (2021).

69. Catena, R., Dopson, S. \& Holweg, M. On the tension between standardized and customized policies in health care: The case of length-of-stay reduction. J. Oper. Manag. 66, 135-150, DOI: https://doi.org/10.1002/joom.1016 (2020).

70. Buijs, P., Catena, R., Holweg, M. \& van der Vaart, T. Preventing disproportionate mortality in icu overload situations: Empirical evidence from the first covid-19 wave in europe. medRxiv DOI: https://doi.org/10.1101/2021.05.03.21255735 (2021).

71. Rocks, S. \& Idriss, O. Did hospital capacity affect mortality during the pandemic's first wave? (2020). https://www.health. org.uk/news-and-comment/charts-and-infographics/did-hospital-capacity-affect-mortality-during-the-pandemic Last accessed on 2021-09-03.

\section{Acknowledgements}

The authors would like to thank the Brazilian Federal Agency for Support and Evaluation of Graduate Education (CAPES) [grant number 88881.133454/2016-01] and Federal Institute of Goias (IFG) [grant numbers: 23378.000781/2017-14 and 23378.000462/2018-81].

\section{Author contributions statement}

V.M.G. and W.P.C. conceived the sensitivity metric; J.R.B.P., V.M.G. and W.P.C. conceived the system complexity metric based on connections; J.R.B.P., V.M.G., P.S.B. and F.R.A. developed the model of the intensive care unit; J.R.B.P. and V.M.G. simulated the systems; J.R.B.P., V.M.G., G.A.W. and F.A.G. analyzed the data. All authors participated effectively in the writing, correction, revision and translation process of this paper.

\section{Additional information}

\section{Competing interests}

The authors declare no competing interests. 